\title{
ERKI/2 is activated in non-small-cell lung cancer and associated with advanced tumours
}

\section{S Vicent ${ }^{1,2}$, JM López-Picazo ${ }^{3}$, G Toledo $^{4}$, MD Lozano ${ }^{4}$, W Torre ${ }^{5}$, C Garcia-Corchón ${ }^{2}$, C Quero ${ }^{3}$, J-C Soria ${ }^{6}$, S Martín-Algarra ${ }^{3}$, RG Manzano ${ }^{1,7}$ and LM Montuenga ${ }^{*, 1,2,7}$}

'Carcinogenesis Unit, Division of Oncology, Centre for Applied Medical Research (CIMA), University of Navarra, Pamplona 31 008, Spain; ${ }^{2}$ Department of Histology and Pathology, University of Navarra, Pamplona 31008, Spain; ${ }^{3}$ Department of Oncology, University Hospital, University of Navarra, Pamplona 31008, Spain; ${ }^{4}$ Department of Pathology, University Hospital, University of Navarra, Pamplona 31008, Spain; ${ }^{5}$ Department of Thoracic Surgery, University Hospital, University of Navarra, Pamplona 31008, Spain; ${ }^{6}$ Department of Medical Oncology, Lung Unit, Institut Gustave Roussy, F-94805 Villejuif, France

Activation of the ERKI/2 pathway is involved in malignant transformation both in vitro and in vivo. Little is known about the role of activated ERKI/2 in non-small cell lung cancer (NSCLC). The purpose of this study was to characterise the extent of the activation of ERKI/2 by immunohistochemistry in patients with NSCLC, and to determine the relationship of ERKI/2 activation with clinicopathological variables. Specimens from I I I patients with NSCLC (stages I-IV) were stained for P-ERK. Staining for epidermal growth factor receptor (EGFR) and Ki-67 was also performed. In all, 34\% of the tumour specimens showed activation for ERKI/2, while normal lung epithelial tissue was consistently negative. There was a strong statistical correlation between nuclear and cytoplasmic P-ERK staining and advanced stages $(P<0.05$ and $P<0.001$, respectively), metastatic hilar or mediastinal lymph nodes $(P<0.01, P<0.00 I)$, and higher $T$ stages $(P<0.01, P<0.00 I)$. We did not find correlation of nuclear or cytoplasmic $P$-ERK staining with either EGFR expression or Ki-67 expression. Total ERKI/2 expression was evaluated with a specific ERKI/2 antibody and showed that P-ERK staining was not due to ERK overexpression but rather to hyperactivation of ERK I/2. Patients with a positive PERK cytoplasmic staining had a significant lower survival $(P<0.05)$. However, multivariate analysis did not show significant survival difference. Our study indicates that nuclear and cytoplasmic ERKI/2 activation positively correlates with stage, T and lymph node metastases, and thus, is associated with advanced and aggressive NSCLC tumours.

British Journal of Cancer (2004) 90, 1047-1052. doi:I0.1038/sj.bjc.660I644 www.bjcancer.com

(c) 2004 Cancer Research UK

Keywords: NSCLC; MAPK; EGFR; Ki-67

In the United States and Europe, lung cancer is the leading cause of cancer death. Non-small cell lung cancer (NSCLC) is the most common type, accounting for $75-80 \%$ of all lung cancers (Dy and Adjei, 2002a). In spite of important advances in surgery, radiation, chemotherapy, and the efforts involved in early detection and prevention, the survival of patients with NSCLC has not changed significantly over the past 20 years (Devesa et al, 1995). This is due to the fact that patients with early stage disease can be effectively treated with surgery, but most patients present at diagnosis with advanced stage, which is essentially incurable, since systemic chemotherapy has poor long-term results in these patients. Even after surgery, $50 \%$ of operated patients will develop metastatic disease (Dy and Adjei, 2002a). All these facts emphasise the need for new early detection tools and for more effective therapies. Our knowledge of the molecular events associated with the biology of

* Correspondence: LM Montuenga, Department of Histology and Pathology, University of Navarra, C/lrunlarrea, Pamplona 31080, Spain; E-mail: Imontuenga@unav.es

${ }^{7}$ These authors contributed equally to the present work

Received II August 2003; revised 13 November 2003; accepted 15 December 2003
NSCLC has substantially increased over the past decade, and extensive molecular and cytogenetic alterations have been characterised in these tumours (Fong et al, 1999). Many molecular mechanisms associated with the pathogenesis of lung cancer offer the exciting possibility of becoming new targets for lung cancer therapy (Dy and Adjei, 2002a, b). A better understanding of the molecular mechanisms involved in lung carcinogenesis and in the progression of this disease is urgently needed to design effective treatments.

One of the signalling pathways that has been extensively studied in vitro is the RAS-RAF-MEK-ERK pathway. Most of the studies carried out to characterise this pathway have been conducted in fibroblasts, although more recently there is also an increasing number of studies in epithelial cell lines (Cobb, 1999). The extracellular signal regulated kinase (ERK1/2) also called the mitogen-activated protein kinase (MAPK), is activated by dual phosphorylation on both Thr202 and Tyr204 residues. This activation is mediated by activated MEK1, which in turn is phosphorylated by RAF-1, and RAF-1 is activated by RAS (for reviews see Cobb, 1999; Widmann et al, 1999; Chang and Karin, 2001). The only known substrates of MEK1 are ERK1 and ERK2, which makes MEK1 an important point to control ERK signalling, and a potential therapeutic target. Activated ERK1 and ERK2 can 
phosphorylate a variety of nuclear substrates such as the transcription factors Elk-1, Ets1 and Sapla, and also cytosolic targets such as phospholipase A2, and S6 Kinase p90 ${ }^{\text {rsk }}$ among others (Widmann et al, 1999). Growth factors such as EGF, HGF and PDGF, as well as a variety of other stimuli activate ERK1/2, and activation of this kinase has been frequently associated with proliferation or differentiation depending on several factors such as cellular context and the length of the activation (Widmann et al, 1999). It is also known that several oncogenes can constitutively activate ERK, and that this uncontrolled activation of ERK can lead to malignant transformation both in vitro and in vivo (Webb et al, 1998). Furthermore, activated ERK1/2 and/or increased levels of ERK1/2 have been reported in a variety of human tumour cell lines (Hoshino et al, 1999) and epithelial cancer tissues such as breast (Sivaraman et al, 1997; Adeyinka et al, 2002), kidney (Oka et al, 1995), colon (Sebolt-Leopold et al, 1999) and head and neck cancers (Albanell et al, 2001). More recently, Blackhall et al (2003) have shown P-ERK activation in small cell lung cancer (SCLC) of a high percentage of patients studied. Although there is some evidence of the presence of activated ERK1/2 in NSCLC primary cancer cells derived from tumours as compared to normal lung cells (Hoshino et al, 1999), the extent of this activation and its meaning and role in patients with NSCLC remain to be defined. Therefore, we analysed the expression of phosphorylated (activated) ERK1/2 by immunohistochemistry with a phosphospecific antibody in a nonselected patient population with histologically proven NSCLC, and made correlations of the expression of this kinase with different clinical parameters and with survival, in an attempt to understand the role of activated ERK1/2 in these patients.

\section{MATERIAL AND METHODS}

\section{Tissue specimens and patient characteristics}

All patients were treated at the University Hospital of the University of Navarra (Clínica Universitaria de Navarra, Pamplona, Spain) from December 1994 to June 2003. Paraffin-embedded tissue specimens from 111 patients were obtained according to institutional review board-approved protocols. These tissue specimens were obtained either from biopsies of the primary tumours corresponding to patients with advanced nonoperable NSCLC or from surgical specimens in those patients who were operated. In both cases, biopsy specimens were obtained prior to any chemo- or radiation therapy treatment. All the samples were fixed in $10 \%$ buffered formalin. Sections $(5 \mu \mathrm{m})$ from the paraffin tissue blocks were processed for immunohistochemical analysis. One section was stained with haematoxylin and eosin (H\&E) and reviewed by at least two pathologists to confirm the presence of NSCLC in the specimen, and also for appropriate histopathological characterisation.

All patients had an ECOG performance status of 0 or 1, adequate baseline organ function (defined as a leucocyte count $>3 \times 10^{9} \mathrm{l}^{-1}$ (absolute granulocyte count $>1.5 \times 10^{9} 1^{-1}$ ), platelet count $>75 \times 10^{9} \mathrm{l}^{-1}$, normal liver function tests and serum creatinine level $<1.4 \mathrm{mg} \mathrm{dl}^{-1}$ ) and no other severe co-morbid conditions. A written signed informed consent was obtained from all patients before treatment.

Patient stage was determined according to the TNM staging system. Surgery was performed as the initial treatment for medically operable stages I, II and IIIA (non-N2 tumours). Lobectomy and ipsilateral mediastinal lymph node sampling was the treatment of choice. Postoperative radiotherapy was added depending on the pathologic findings. Specifically, radiation therapy was recommended with pathologically confirmed metastatic hilar or mediastinal lymph nodes. Postoperative radiotherapy consisted of 45 grays (Gy) using conventional fields and fractionation schedules.
Patients with locally advanced NSCLC were initially treated with chemotherapy. Intravenous infusions of paclitaxel $135 \mathrm{mg} \mathrm{m}^{-2}$ over $3 \mathrm{~h}$ and cisplatin $120 \mathrm{mg} \mathrm{m}^{-2}$ over $6 \mathrm{~h}$ were delivered on day 1 . Either vinorelbine $30 \mathrm{mg} \mathrm{m}^{-2}$ or gemcitabine $800 \mathrm{mg} \mathrm{m}^{-2}$ over 30 min was administered on days 1 and 8 . Cycles were repeated every 4 weeks. Four cycles were delivered to responding patients before administering radical radiotherapy with concurrent chemotherapy. Patients who did not respond to this treatment were administered radical hyperfractionated radiotherapy $(69.6 \mathrm{~Gy})$ soon after progression to chemotherapy was detected. Stage IV patients were treated with the chemotherapy schedule previously described for stage III patients. Median follow-up time of the whole group was 30 months (range $1-87$ ).

Clinicopathological characteristics including age, gender, histology, stage and grade of differentiation were evaluated. We also evaluated the expression of the epidermal growth factor receptor (EGFR) and the nuclear antigen Ki-67 by immunohistochemistry in order to assess whether there was any correlation between activation of ERK1/2 and the expression of EGFR or the proliferation.

\section{Immunohistochemistry}

Samples from 111 patients were studied by immunohistochemistry. Rabbit polyclonal antibodies specific against the dual phosphorylated form of ERK1/2 (Thr202/Tyr204) were used (Cell Signaling, Beverly, MA, USA) at a dilution of 1:100 (determined after preliminary serial dilution studies). Samples were incubated overnight at $4{ }^{\circ} \mathrm{C}$ with the primary antibody. Immunocytochemical reaction was shown using the EnVision ${ }^{\circledR}$ TM intensifying kit (Dako, Carpinteria, CA, USA). Positive controls were ERK1/2activated HTB58 human lung cancer cells fixed in $10 \%$ buffered formalin, which were analysed by immunohistochemistry with the anti-P-ERK polyclonal antibody. Activation of ERK1/2 was achieved by culturing the cells in a medium containing $20 \%$ fetal calf serum (FCS) after $16 \mathrm{~h}$ of serum deprivation (medium with $0.4 \%$ FCS) (data not shown). We also performed Western blot analyses of the induction of P-ERK after treatment of the cells with two known activating agents, fetal bovine serum $(20 \%)$ and TPA $\left(100 \mathrm{ng} \mathrm{ml}^{-1}\right)$ (data not shown). Negative controls consisted of incubation of serial (positive) lung tumour sections with an unrelated IgG rabbit polyclonal antibody. The results of the staining were reviewed independently without knowledge of the clinicopathological data by three of the co-authors (SVC, CGC and LMM) who scored the percentage of positive nuclei and/or cytoplasms within tumours and in the normal matching lung at high magnification. Average of the scores was registered and no significant divergence was found in the scoring among the observers. Serial cuts $(3 \mu \mathrm{m})$ of a subset of the specimens (five samples that had high percentage of ERK1/2 activation and five samples that had no activated ERK1/2) were stained using a specific antibody against total ERK1/2 (Cell Signaling, Beverly, MA, USA). This experiment was also carried out using a dilution of $1: 100$ as described for the P-ERK antibody. The median percentage of P-ERK stained cells was $15 \%$ and this was chosen as the cutoff value. A score of $15 \%$ or higher was considered positive. Intensity of the staining was also registered and evaluated in a semiquantitative way but the intensity of the staining was fairly similar from specimen to specimen, and its inclusion in the statistical analysis did not modify the calculations based on extension alone; therefore, it will not be reported here.

The epidermal growth factor receptor and Ki-67 primary antibodies were from Novocastra (Newcatle, UK) and were used at $1: 20$ and $1: 100$ dilutions, respectively. They both were used according to the manufacturer's recommendations, including the use of appropriate positive and negative controls for each antibody. For the EGFR staining, three of the authors (GT, CGC and LMM) scored the specimens as positive, when any intensity 
and extension of staining was apparent, or negative when no signs of staining at all were seen, as described by other authors (Albanell et al, 2001). A positive score for the Ki-67 staining was considered when more than $5 \%$ of the tumour nuclei were stained as described before (Xu et al, 2002).

\section{Statistical analysis}

All statistical methods used were carried out using the statistical package SPSS Data Analysis Program version 9.0. The methods used were the Fisher's exact test, the $\chi^{2}$ test, the Mann - Whitney $U$ test, the Kaplan-Meier log rank test and the Cox regression test for univariate and multivariate survival analysis.

\section{RESULTS}

A total of 111 patients with histologically proven NSCLC were evaluated in this study. The characteristics of these patients are shown in Table 1. The normal adjacent tissue in 30 of the samples was also studied. Normal lung tissue was negative for ERK1/2 activation in all the 30 samples studied. Specifically, type I and II alveolar pneumocytes did not show any staining for P-ERK, and bronchiolar epithelium was also negative for P-ERK (Figure 1A and B). Endothelial cells were also negative for P-ERK staining in the histologically normal areas.

Immunohistochemical staining for P-ERK in the tumours showed that a variable percentage of cells was stained in each sample and that the staining could be located in the nucleus or in the cytoplasm, but most often it was present in both nucleus and cytoplasm, usually with a predominant staining in the latter. We recorded and analysed the staining for P-ERK according to its intracellular location. Examples of positive and negative staining are shown in Figure 1C-F. For statistical purpose, patients were

Table I Characteristics of the patients

\begin{tabular}{|c|c|c|}
\hline & No. of patients $(n=I I I)$ & $\%$ Total \\
\hline \multicolumn{3}{|l|}{ Age } \\
\hline Median & 62 & \\
\hline Range & $31-87$ & \\
\hline \multicolumn{3}{|l|}{ Gender } \\
\hline Male & 93 & 83.7 \\
\hline Female & 18 & 16.3 \\
\hline \multicolumn{3}{|l|}{ Histology } \\
\hline$A C$ & 50 & 46 \\
\hline SC & 57 & 51 \\
\hline Mixt & 3 & 3 \\
\hline \multicolumn{3}{|l|}{$T$} \\
\hline । & 36 & 32.5 \\
\hline 2 & 48 & 43.2 \\
\hline 3 & 12 & 10.8 \\
\hline 4 & 11 & 9.9 \\
\hline$x$ & 3 & 2.7 \\
\hline \multicolumn{3}{|l|}{ Grade } \\
\hline I & 9 & 13 \\
\hline 2 & 28 & 40.6 \\
\hline 3 & 32 & 46.4 \\
\hline \multicolumn{3}{|l|}{ N } \\
\hline 0 & 73 & 65.8 \\
\hline 1 & 15 & 13.5 \\
\hline 2 & 16 & |4.4 \\
\hline 3 & 7 & 6.3 \\
\hline \multicolumn{3}{|l|}{ Stage } \\
\hline 1 & 62 & 55.9 \\
\hline$\|$ & 18 & 16.2 \\
\hline III & 28 & 25.2 \\
\hline IV & 3 & 2.7 \\
\hline
\end{tabular}

arranged in two groups: (a) patients with less than $15 \%$ of tumour cells stained for P-ERK and (b) patients with $15 \%$ or more cells stained. Using this cutoff, specimens were also scored according to the location of the staining: nuclear staining (with or without accompanying cytoplasmic staining) or cytoplasmic staining (with or without accompanying nuclear staining). Although not reported, the results obtained shifting the cutoff values from 510 to $20-25 \%$ had a similar trend to those shown here using the cutoff value of $15 \%$. Overall, $65.8 \%$ of tumors were negative and $34.2 \%$ positive when nuclear and cytoplasmic staining was considered. Among positive NSCLC, at least half of the tumours showed ERK1/2 activation preferentially located at the periphery of the tumour.

We found a strong statistical correlation between a positive nuclear and cytoplasmic P-ERK staining and advanced stages $(P=0.04, P=0.001)$, higher T stages (T1-2 vs T3-4; $P=0.005$, $P=0.001$ ), and the presence of metastatic lymph nodes (N0 vs N13; $P=0.001, P=0.001$ ). However, there was no statistically significant difference between patients whose tumours had negative staining $v s$ those patients whose tumours had positive staining either in the nucleus or in the cytoplasm when comparing age, sex and histology (squamous cell carcinoma $v s$ adenocarcinoma) (Table 2). The grade of differentiation was only associated with cytoplasmic P-ERK staining but not with nuclear staining (1 and 2 vs $3, P=0.046$ for cytoplasmic staining and $P=0.271$ for nuclear staining).

Staining with a specific total ERK1/2 antibody of a subset of 10 tumour specimens (five negative and five positive for P-ERK staining) showed the presence of a homogeneous staining for total
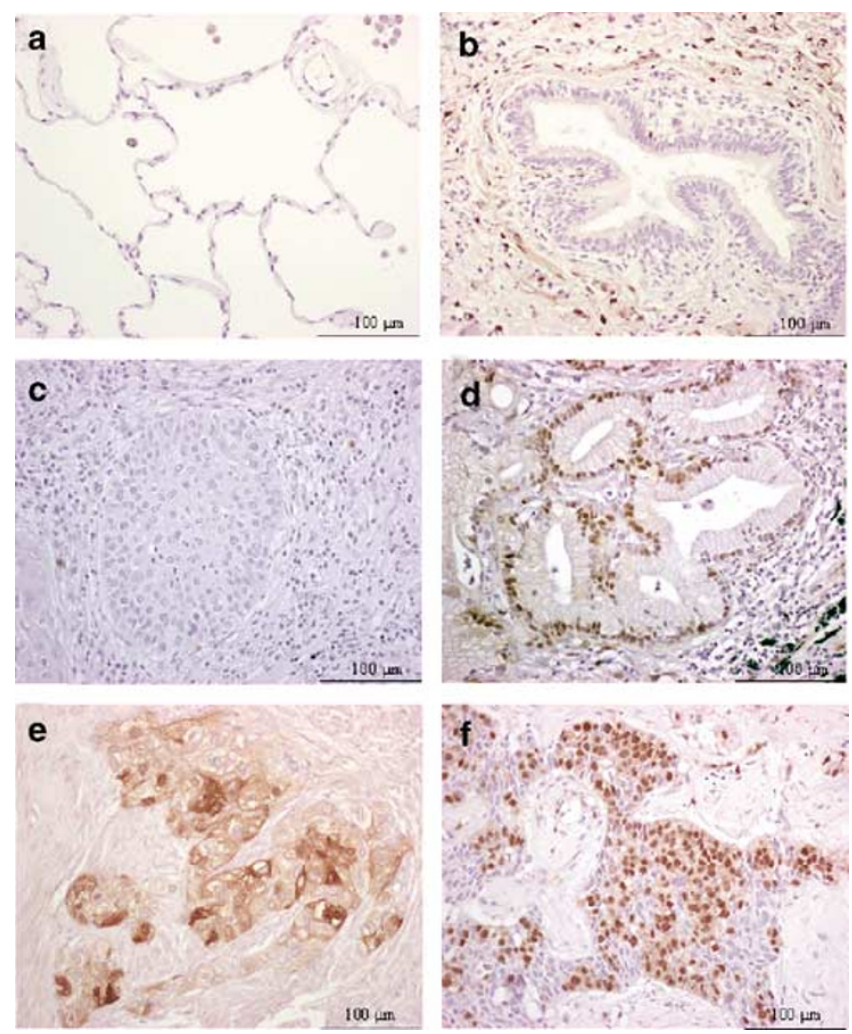

Figure I Immunohistochemical staining of normal lung and NSCLC specimens with a specific antibody against P-ERK: (A) normal alveolar epithelium, negative staining; (B) normal bronchiolar epithelium, negative staining; (C) squamous cell carcinoma, negative staining; (D) adenocarcinoma with nuclear P-ERK staining; (E) squamous cell carcinoma with cytoplasmic and moderate nuclear staining; (F) adenocarcinoma with nuclear and extensive cytoplasmic staining. 
ERK1/2 in the 10 specimens independently of their P-ERK status. These results suggest that the expression of P-ERK is not the consequence of overexpression of ERK1/2 but rather the result of hyperactivation of ERK1/2 in the specimens.

Trying to understand whether there is any relationship between the presence of activated ERK1/2 and the EGF-dependent growth regulatory pathway in NSCLC, staining of the same samples for EGFR was carried out. In some systems, activated ERK1/2 correlates well with proliferation. Thus, immunohistochemical staining for Ki-67 (as a surrogate marker of cell proliferation) was also performed in this series of tumours. Neither EGFR nor Ki-67 expression correlates in the specimens analysed with activated ERK1/2 (cytoplasmic or nuclear) as detected by immunohistochemistry (Table 2).

Univariate analysis of clinicopathological factors relevant to patient survival showed that the following factors studied were statistically significant for the survival of patients: stage $(P=0.001), \mathrm{T}(P=0.001), \mathrm{N}(P=0.001)$ and a positive cytoplasmic $\mathrm{P}$-ERK staining $(P=0.03)$. Interestingly, the presence of nuclear $\mathrm{P}$ ERK staining was not statistically significant $(P=0.509)$. Figure 2 shows a Kaplan-Meier plot of patients whose tumours had positive cytoplasmic staining (15\% or more cells with immunostained cytoplasm) and those whose tumours had negative P-ERK staining (less than $15 \%$ of cells with immunostained cytoplasm). This plot shows that the median survival of patients with positive P-ERK staining is 57 months (95\% confidence interval 11-102 months), whereas for patients with negative staining the median survival has not been reached, and this difference is statistically

Table 2 Clinicopathological factors and their relationship to the expression of the different proteins assessed

\begin{tabular}{lcccc}
\hline & $\begin{array}{c}\text { P-ERK } \\
\text { nuclear }\end{array}$ & $\begin{array}{c}\text { P-ERK } \\
\text { cytoplasmic }\end{array}$ & Ki-67 & EGFR \\
\hline Age & $P=0.7$ & $P=0.115$ & $P=0.277$ & $P=0.143$ \\
Sex & $P=0.349$ & $P=1$ & $P=0.058$ & $P=0.146$ \\
Histology & $P=0.812$ & $P=0.65$ & $\mathbf{P}=\mathbf{0 . 0 4}$ & $P=0.28$ \\
Grade & $P=0.271$ & $\mathbf{P}=\mathbf{0 . 0 4 6}$ & $P=0.653$ & $P=0.54$ \\
Stage & $\mathbf{P}=\mathbf{0 . 0 4}$ & $\mathbf{P}=\mathbf{0 . 0 0 0}$ & $P=0.902$ & $P=0.3$ \\
T & $\mathbf{P}=\mathbf{0 . 0 0 5}$ & $\mathbf{P}=\mathbf{0 . 0 0 0}$ & $P=0.586$ & $P=0.58$ \\
N & $\mathbf{P}=\mathbf{0 . 0 0 1}$ & $\mathbf{P}=\mathbf{0 . 0 0 0}$ & $P=0.897$ & $P=0.64$ \\
EGFR & $P=0.289$ & $P=0.63$ & $P=0.096$ & \\
Ki-67 & $P=0.211$ & $P=0.424$ & & \\
\hline
\end{tabular}

Bold type values indicate significance at levels $<0.05$. $P$, probability value.

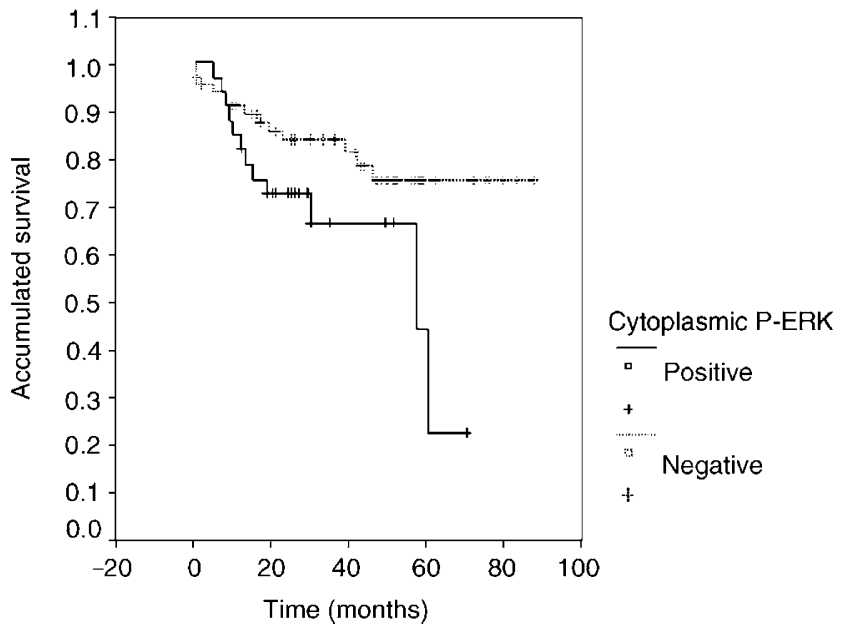

Figure 2 Kaplan-Meier survival plot by cytoplasmic P-ERK expression ( $15 \%$ cutoff). Survival plot of all patients. significant with $P=0.02$. The estimated 5-year survival rate was $43 \%$ for patients with positive P-ERK staining $v s 77 \%$ for patients whose tumours had negative staining for P-ERK. However, survival analysis of the NSCLC patients analysed in this study did not show any significant differences according to the expression of either EGFR $(P=0.18)$ or Ki-67 $(P=0.13)$.

In order to determine whether P-ERK staining is an independent prognostic factor in NSCLC, as it is strongly associated with advanced stages and metastatic tumours, we performed a multivariate analysis by adjusting phospho-ERK1/2 activation to clinical stage (a parameter that depends on $\mathrm{T}$ and $\mathrm{N}$ ), which influences patient survival. The test showed that patients with negative P-ERK did not live significantly longer than patients with positive P-ERK $(P=0.69)$.

\section{DISCUSSION}

Although an increased amount of information has been gathered on the role of the activation of ERK1/2 in a variety of cell lines, very little is known about its potential role in human cancer. A few previous reports tried to address this issue by studying the degree of activation of ERK1/2 in different human tumours. Oka et al (1995) showed that there was a constitutive activation of ERK1/2 in $48 \%$ of renal cancer samples (12 out of 25 patients) when compared to normal kidney tissue, suggesting that this signalling pathway could play a role in renal cancer. It was also reported that activated ERK1/2 was present in 58\% of hepatocellular carcinomas studied, and that this activation was higher than in adjacent noncancerous lesions (Ito et al, 1998). Another report found no ERK1/2 activation in normal prostatic tissues and an increased expression of activated ERK1/2 in prostatic carcinoma tissues in advanced stages and in recurrent cases (Gioeli et al, 1999). There was also evidence of activated ERK1/2 in patients with glioma (Mandell et al, 1998), breast cancer (Sivaraman et al, 1997; Adeyinka et al, 2002), head and neck cancer (Albanell et al, 2001) and melanoma (Cohen et al, 2002). In our study, we show for the first time that normal matching lung tissue in the tumour specimens analysed, as previously reported for other tumour types, does not show activated ERK1/2, while activation of ERK1/2 is frequently found in NSCLC. Little evidence existed regarding the expression of activated ERK1/2 in lung cancer and, specially, in NSCLC. A previous report showed increased levels of activated ERK1/2 in a very reduced number of lung cancer tissues when compared to matching normal lung (Hoshino et al, 1999). Recently, Blackhall et al (2003) described that ERK1/2 is activated in SCLC, and Mukohara et al (2003) have studied some elements of the EGFR signalling pathway in a much smaller number of NSCLC patients. We have also found that a high percentage of NSCLC patients (38 out of 111) present ERK1/2 activation. In this regard, we have seen that a high percentage of positive NSCLC specimens $(50 \%)$ presented ERK1/2 activation at the periphery of the tumour. This pattern of staining had been described by Albanell et al (2001) in head and neck cancers and also by Mukohara et al (2003) in NSCLC. Therefore, it seems that activated ERK1/2 is a landmark of a subset of NSCLC.

In the present work, we used phosphospecific antibodies to identify activated ERK1/2. The antibody used in this study is the same antibody used in most of the immunohistochemistry studies performed to date (Sivaraman et al, 1997; Mandell et al, 1998; Albanell et al, 2001; Adeyinka et al, 2002; Cohen et al, 2002). Besides, this antibody was validated in our lab specifically for formaline-fixed tissues (data not shown). There are other methods to assess the phospho-ERK status in patient specimens, such as Western blot. This technique has the inconvenience of requiring a larger amount of tissue, and that the inclusion of adjacent and stromal tissues along with the tumour in the extracts may mask the real activation status of ERK1/2. 
To our knowledge, only a few studies published to date provide data on the clinical outcome of patients with activated ERK1/2. In breast cancer patients, phosphorylated ERK1/2 (as determined by immunohistochemistry) may predict a poor response to hormonal therapy and, in a subset of patients, a worse survival (Mueller et al, 2000; Gee et al, 2001). In SCLC, and regarding cytoplasmic staining of phospho-ERK1/2, the activation of this MAPK positively correlates with survival. In our work, we have seen a strong correlation of activated ERK1/2 with some clinicopathological variables. In particular, we have demonstrated that there is a strong correlation between positive nuclear and cytoplasmic staining for P-ERK and more aggressive tumours (i.e. advanced stage tumours (III and IV), tumours with hilar or mediastinal lymph node metastases, and tumours with higher $\mathrm{T}$ stage), and also between cytoplasmic staining and the grade of differentiation. With regard to survival, although we have seen in invariable analysis that P-ERK cytoplasmic expression may predict poor survival, multivariate analysis did not confirm that cytoplasmic ERK activation could be used as an independent prognostic factor. This may be due to the strong association of P-ERK with advanced stage tumours, metastatic tumours and tumours with higher $\mathrm{T}$ stage. In NSCLC, a recent report on the expression of EGFR and downstream-activated signalling factors activated by this receptor (Mukohara et al, 2003) showed that P-ERK is activated in about one-third of NSCLC, as we report in the present study in a much larger series. In contrast to our data, they found no correlation of the activation of this MAPK with clinicopathological variables. Another difference in our results with respect to those published by Mukohara et al (2003) is that we have almost the double number of patients $(n=111$ vs $N=60)$. This gives more statistical support and confidence to the results obtained in this study. Besides, we have used an antibody that is different from the one used by Mukohara's group. We have validated the specificity of the antibody by specific experiments and by its utilisation in other studies by previous authors (Sivaraman et al, 1997; Mandell et al, 1998; Albanell et al, 2001; Adeyinka et al, 2002; Cohen et al, 2002). These two differences may explain the divergent results between our studies in relation to phospho-ERK and its correlation with clinicopathological variables.

A previous report showed that in head and neck squamous cancer, activated ERK1/2 correlated with the expression of the EGFR (Albanell et al, 2001). In our patients we also looked at the correlation between EGFR and the expression of activated ERK1/2, but we did not find such a correlation. It is quite likely that activation of the EGFR, rather than merely its expression, is what is relevant in terms of activation of ERK. If this is the case, it could also be of interest to determine the levels of phospho-EGFR in addition to the levels of activated ERK1/2 in patients with NSCLC to test whether patients with a positive staining could benefit from specific inhibitors of EGFR, like Iressa. In fact, both the phosphorylated EGFR and P-ERK specific antibodies have already been used to study the pharmacodynamic effects of Iressa in skin biopsies of patients treated with the drug (Albanell et al, 2002). Our results may suggest a greater involvement of other growth factors in the activation of the ERK1/2 signalling pathway and, therefore, in the progression of NSCLC. As an example, a potential role of FGF in NSCLC has already been reported (Berger et al, 1999; Guddo et al, 1999).

\section{REFERENCES}

Adeyinka A, Nui Y, Cherlet T, Snell L, Watson PH, Murphy LC (2002) Activated mitogen-activated protein kinase expression during human breast tumorigenesis and breast cancer progression. Clin Cancer Res 8: $1747-1753$

Albanell J, Codony-Servat J, Rojo F, Del Campo JM, Sauleda S, Anido J, Raspall G, Giralt J, Rosello J, Nicholson RI,
Our data also suggest the relevance of the intracellular location of the immunostaining for P-ERK. In SCLC, Blackhall et al (2003) had reported that cytoplasmic and not nuclear phospho-ERK staining correlated with patients survival. In our work, we have shown that only the cytoplasmic staining was associated with a poorer survival in NSCLC. As ERK1/2 has both nuclear and cytoplasmic substrates (Widmann et al, 1999), this observation could indicate an important role of cytoplasmic substrates of ERK1/2 in advanced and metastatic tumours. Recently, more cytoplasmic substrates of phospho-ERK1/2 involved in apoptosis (such as caspase 9) have been described, indicating that ERK1/2 activation directly regulates programmed cell death (Allan et al, 2003). In lung cancer, although there is some indication of the possible contribution of some of the nuclear substrates of ERK1/2 to the biology and prognosis of NSCLC patients (Levin et al, 1995; Volm et al, 2002), very little is known about the possible role of cytoplasmic substrates of ERK1/2 in NSCLC.

Another potential implication of our findings is related to a new class of therapeutic agents: the MEK1 inhibitors. Although inhibitors of MEK1, like PD98059 (Alessi et al, 1995; Dudley et al, 1995) and U0126 (Duncia et al, 1998), have been used for quite a while in laboratory studies, it was not until recently that the first compound of this new class of drugs, called PD184352 (SeboltLeopold et al, 1999), entered clinical trials. Previous MEK1 inhibitors lacked solubility, which prevented their use in the clinical setting. Preliminary results of phase I trials with PD184352 have shown encouraging results, and a clinical trial of this agent in patients with NSCLC has already started (Dy and Adjei, 2002a). Monitoring the P-ERK status in biopsies of NSCLC patients entering clinical trials of the newly introduced inhibitors of MEK1 will help to assess whether an activated ERK1/2 pathway is necessary for a MEK1 inhibitor to be effective, a question that at present remains unanswered.

In summary, we have shown that the detection of immunoreactivity for P-ERK in patients with NSCLC is associated with advanced and aggressive tumours. Our data also suggest that the analysis of ERK1/2 activation may be useful to identify a subgroup of patients with a poorer prognosis. Finally, our results suggest that the relevance of P-ERK might not be related to the proliferation rate of NSCLC but rather to other tumour characteristics such as the resistance to undergo apoptosis. In this regard, the role of ERK1/2 in cell survival and regulation of apoptosis is well established in various systems (Xia et al, 1995; Mackeigan et al, 2000). Besides, in lung cancer, Brognard and Dennis (2002) have pointed out the importance of ERK1/2 activation in NSCLC cell lines, where it promotes cell survival and chemotherapeutic resistance. Further studies are warranted to explore the role of activated ERK1/2 in patients with NSCLC.

\section{ACKNOWLEDGEMENTS}

We acknowledge the Department of Education at the Government of Navarra and 'Fundacion Jose y Ana Royo' for their partial funding of this work. This work was also funded by the Spanish Ministry of Health (RTICC 10/03) and through the agreement between FIMA and the "UTE project CIMA".
Mendelsohn J, Baselga J (2001) Activated extracellular signal-regulated kinases: association with epidermal growth factor receptor/transforming growth factor alpha expression in head and neck squamous carcinoma and inhibition by anti-epidermal growth factor receptor treatments. Cancer Res 61: $6500-6510$ 
Albanell J, Rojo F, Averbuch S, Feyereislova A, Mascaro JM, Herbst R, LoRusso P, Rischin D, Sauleda S, Gee J, Nicholson RI, Baselga J (2002) Pharmacodynamic studies of the epidermal growth factor receptor inhibitor ZD1839 in skin from cancer patients: histopathologic and molecular consequences of receptor inhibition. J Clin Oncol 20: $110-124$

Alessi DR, Cuenda A, Cohen P, Dudley DT, Saltiel AR (1995) PD 098059 is a specific inhibitor of the activation of mitogen activated protein kinase kinase in vitro and in vivo. J Biol Chem 270: 27489-27494

Allan LA, Morrice N, Brady S, Magee G, Pathak S, Clarke PR (2003) Inhibition of caspase- 9 through phosphorylation at Thr 125 by ERK MAPK. Nat Cell Biol 7: 647-654

Berger W, Setinek U, Mohr T, Kindas-Mugge I, Vetterlein M, Dekan G, Eckersberger F, Caldas C, Micksche M (1999) Evidence for a role of FGF2 and FGF receptors in the proliferation of non-small cell lung cancer cells. Int J Cancer 83: 415-423

Blackhall FH, Pintilie M, Michael M, Leighl N, Feld R, Tsao MS, Shepherd FA (2003) Expression and prognostic significance of kit, protein kinase $\mathrm{B}$, and mitogen-activated protein kinase in patients with small cell lung cancer. Clin Cancer Res 9: 2241-2247

Brognard J, Dennis P (2002) Variable apoptotic response of NSCLC cells to inhibition of the MEK/ERK pathway by small molecules or dominant negative mutants. Cell Death Differ 9: 893-904

Chang L, Karin M (2001) Mammalian MAP kinase signalling cascades. Nature 410: $37-40$

Cobb MH (1999) MAP kinase pathways. Prog Biophys Mol Biol 71: 479-500

Cohen CG, Zavala-Pompa A, Sequeira JH, Shoji M, Sexton DG, Cotsonis G, Cerimele F, Govindarajan B, Macaron N, Arbiser JL (2002) Mitogen-actived protein kinase activation is an early event in melanoma progression. Clin Cancer Res 8: 3728 - 3733

Devesa SS, Blot WJ, Stone BJ, Miller BA, Tarone RE, Fraumeni Jr JF (1995) Recent cancer trends in the United States. J Natl Cancer Inst 87: 175 - 182

Dudley DT, Pang L, Decker SJ, Bridges AJ, Saltiel AR (1995) A synthetic inhibitor of the mitogen-activated protein kinase cascade. Proc Natl Acad Sci USA 92: 7686 - 7689

Duncia JV, Santella III JB, Higley CA, Pitts WJ, Wityak J, Frietze WE, Rankin FW, Sun JH, Earl RA, Tabaka AC, Teleha CA, Blom KF, Favata MF, Manos EJ, Daulerio AJ, Stradley DA, Horiuchi K, Copeland RA, Scherle PA, Trzaskos JM, Magolda RL, Trainor GL, Wexler RR, Hobbs FW, Olson RE (1998) MEK inhibitors: the chemistry and biological activity of U0126, its analogs, and cyclization products. Bioorg Med Chem Lett 8: $2839-2844$

Dy GK, Adjei AA (2002a) Novel targets for lung cancer therapy: part I. J Clin Oncol 20: $2881-2894$

Dy GK, Adjei AA (2002b) Novel targets for lung cancer therapy: part II. $J$ Clin Oncol 20: 3016-3028

Fong KM, Sekido Y, Minna JD (1999) Molecular pathogenesis of lung cancer. I Thorac Cardiovasc Surg 118: 1136-1152

Gee JM, Robertson JF, Ellis IO, Nicholson RI (2001) Phosphorylation of ERK1/2 mitogen-activated protein kinase is associated with poor response to anti-hormonal therapy and decreased patient survival in clinical breast cancer. Int J Cancer 95: 247-254

Gioeli D, Mandell JW, Petroni GR, Frierson Jr HF, Weber MJ (1999) Activation of mitogen-activated protein kinase associated with prostate cancer progression. Cancer Res 59: 279-284

Guddo F, Fontanini G, Reina C, Vignola AM, Angeletti A, Bonsignore G (1999) The expression of basic fibroblast growth factor (bFGF) in tumor- associated stromal cells and vessels is inversely correlated with non-small cell lung cancer progression. Hum Pathol 30: 788-794

Hoshino R, Chatani Y, Yamori T, Tsuruo T, Oka H, Yoshida O, Shimada Y, Ari-i S, Wada H, Fujimoto J, Kohno M (1999) Constitutive activation of the $41-/ 43-\mathrm{kDa}$ mitogen-activated protein kinase signaling pathway in human tumors. Oncogene 18: 813-822

Ito Y, Sasaki Y, Horimoto M, Wada S, Tanaka Y, Kasahara A, Ueki T, Hirano T, Yamamoto H, Fujimoto J, Okamoto E, Hayashi N, Hori M (1998) Activation of mitogen-activated protein kinases/extracellular signal-regulated kinases in human hepatocellular carcinoma. Hepatology 27: $951-958$

Levin WJ, Press MF, Gaynor RB, Sukhatme VP, Boone TC, Reissmann PT, Figlin RA, Holmes EC, Souza LM, Slamon DJ (1995) Expression patterns of immediate early transcription factors in human non-small cell lung cancer. The Lung Cancer Study Group. Oncogene 11: $1261-1269$

Mackeigan JP, Collins TS, Ting JP (2000) MEK inhibition enhances paclitaxel-induced tumor apoptosis. J Biol Chem 275: $38953-38956$

Mandell JW, Hussaini IM, Zecevic M, Weber MJ, VandenBerg SR (1998) In situ visualization of intratumor growth factor signaling: immunohistochemical localization of activated ERK/MAP kinase in glial neoplasms. Am J Pathol 153: 1411 - 1423

Mueller H, Flury N, Eppenberger-Castori S, Kueng W, David F, Eppenberger U (2000) Potential prognostic value of mitogen-activated protein kinase activity for disease-free survival of primary breast cancer patients. Int J Cancer 89: $384-388$

Mukohara T, Kudoh S, Yamauchi S, Kimura T, Yoshimura N, Kanazawa H Hirata K, Wanibuchi H, Fukushima S, Inoue K, Yoshikawa J (2003) Expression of epidermal growth factor receptor (EGFR) and downstream-activated peptides in surgically excised non-small cell lung cancer. Lung Cancer 41: 123-130

Oka H, Chatani Y, Hoshino R, Ogawa O, Kakehi Y, Terachi T, Okada Y, Kawaichi M, Kohno M, Yoshida O (1995) Constitutive activation of mitogen-activated protein (MAP) kinases in human renal cell carcinoma. Cancer Res 55: 4182-4187

Sebolt-Leopold JS, Dudley DT, Herrera R, Van Becelaere K, Wiland A, Gowan RC, Tecle H, Barrett SD, Bridges A, Przybranowski S, Leopold WR, Saltiel AR (1999) Blockade of the MAP kinase pathway suppresses growth of colon tumors in vivo. Nat Med 5: 810-816

Sivaraman VS, Wang H, Nuovo GJ, Malbon CC (1997) Hyperexpression of mitogen-activated protein kinase in human breast cancer. J Clin Invest 99: $1478-1483$

Volm M, Koomagi R, Mattern J, Efferth T (2002) Expression profile of genes in non-small cell lung carcinomas from long-term surviving patients. Clin Cancer Res 8: $1843-1848$

Webb CP, Van Aelst L, Wigler MH, Woude GF (1998) Signaling pathways in Ras-mediated tumorigenicity and metastasis. Proc Natl Acad Sci USA 95: $8773-8778$

Widmann C, Gibson S, Jarpe MB, Johnson GL. (1999) Mitogen-activated protein kinase: conservation of a three-kinase module from yeast to human. Physiol Rev 79: $143-180$

Xia Z, Dickens M, Raingeaud J, Davis RJ, Greenberg ME (1995) Opposing effects of ERK and JNK-p38 MAP kinases on apoptosis. Science 270: $1326-1331$

Xu JL, Lai R, Kinoshita T, Nakashima N, Nagasaka T (2002) Proliferation, apoptosis, and intratumoral vascularity in multiple myeloma: correlation with the clinical stage and cytological grade. J Clin Pathol 55: 530-534 